\title{
Atributos químicos de latossolos amarelos em áreas de ocorrência da Soja Louca II na Região Oeste do Pará
}

A dinâmica dos nutrientes no solo é resultado da interação de vários fatores, e, no SPD, os mecanismos que governam são modificados em velocidade e/ou intensidade pela redução do revolvimento do solo e manutenção dos resíduos culturais na superfície do solo. Anomalias em plantas relacionadas a solo são diretamente ligadas aos níveis críticos e tóxicos dos atributos físico, químico ou biológicos de solo, logo, a fertilidade do solo é um dos fatores de predisposição das plantas aos patógenos. A soja louca-II é uma anomalia que causa cerda $40 \%$ perdas em lavouras e sem causa definida. Objetivo do foi avaliar os teores e variância dos atributos químicos de solos em áreas de com ocorrência da Soja Louca Il. Realizaram-se 180 amostras em $20 \mathrm{~cm}, 40 \mathrm{~cm}$ e $60 \mathrm{~cm}$ de profundidas em áreas com e sem a ocorrência da anomalia em propriedades nos municípios de Santarém, Belterra e Mojui dos Campos na região oeste do Pará Os Atributos químicos Ph, P, K, Na, Ca, Mg, Al, Fe, Cu, Zn, Mn e CTC e Teor de Argila natural foram submetidos a análise descritiva e análise de variâncias no programa Biostat versão 5.3. Não foram encontras diferenças significativas entre os atributos nos tratamentos, sendo consideradas estatisticamente semelhantes as áreas com e sem ocorrência da Soja Louca II, porem com terrores de micronutriente em níveis tóxicos na maior parte das amostras em ambos os tratamentos em todas as profundidades coletadas. Os atributos que apresentaram maior e menor Coeficiente de Variação foram pH $(7.45 \%)$ e $\mathrm{Zn}(198,17 \%)$ a $20 \mathrm{~cm}, \mathrm{pH}(6,66 \%)$ e $\mathrm{Cu}$ $(250,70 \%)$ a $40 \mathrm{~cm}$ e pH $(5,84 \%)$ e $\mathrm{Cu}(238,74 \%)$ a $60 \mathrm{~m}$ de profundidade. A análise de variância dos atributos químicos não apresentou diferenças significativa entre as profundidades.

Palavras-chave: Fertilidade do solo; Interação solo-planta; Fitotoxidez.

\section{Chemical attributes of yellow latosols in areas of occurrence of Mad Soya II in the West Region of Pará}

The efficiency of nutrients in the soil is the result of the interaction of several factors, and, in the SPD, the governing mechanisms are modified in speed and/or intensity by reducing soil disturbance and maintaining cultural foods on the soil surface. Anomalies in soil-related plants are directly linked to critical and toxic levels of physical, chemical or biological soil substances, logo, soil fertility and one of the factors of plant predisposition to pathogens. Mad soy-II is an anomaly that causes $40 \%$ reduction in crops and without a definite cause. The objective was to evaluate the contents and the variation of the chemical attributes of the soils in areas of occurrence of Soja Louca II. 180 colors are performed in $20 \mathrm{~cm}, 40 \mathrm{~cm}$ and $60 \mathrm{~cm}$ depth in areas with and without occurrence of anomalies in properties in the municipalities of Santarém, Belterra and Mojui of the fields in western Pará The chemical attributes $\mathrm{Ph}, \mathrm{P}, \mathrm{K}, \mathrm{Na}, \mathrm{Ca}, \mathrm{Mg}, \mathrm{Al}, \mathrm{Fe}, \mathrm{Cu}, \mathrm{Zn}, \mathrm{Mn}$ and CTC and Natural Clay Content were subjected to descriptive analysis and analysis of variances in the Biostat version 5.3 program. No differentiated differences were found between the attributes in the controls, being used statistically as areas with and without occurrence of Crazy Soy II, due to micronutrient earthquakes at toxic levels in most samples in both tests at all depths collected. The attributes with the highest and lowest variation coefficient were $\mathrm{pH}(7,45 \%)$ and $\mathrm{Zn}$ $(198,17 \%)$ at $20 \mathrm{~cm}, \mathrm{pH}(6,66 \%)$ and $\mathrm{Cu}(250,70 \%)$ at $40 \mathrm{~cm}$ and $\mathrm{pH}(5,84 \%)$ and $\mathrm{Cu}(238,74 \%)$ at $60 \mathrm{~m}$ depth. An analysis of variation in chemical attributes did not show significant differences between depths.

Keywords: Soil fertility; Soil-plant interaction; Phytotoxicity.

\section{Topic: Ciências do Solo}

Reviewed anonymously in the process of blind peer.
Received: 02/10/2020

Approved: 20/11/2020
Avner Brasileiro dos Santos Gaspar (iD)

Universidade Federal do Oeste do Pará, Brasil

http://lattes.cnpq.br/1906582047383770

http://orcid.org/0000-0002-6012-852X

avnergaspar@gmail.com

Rodrigo da Silva (iD

Universidade Federal do Oeste do Pará, Brasil

http://orcid.org/0000-0001-9222-5861

rsilvf@gmail.com
Referencing this:

GASPAR, A. B. S.; SILVA, R.. Atributos químicos de latossolos amarelos em áreas de ocorrência da Soja Louca II na Região Oeste do Pará. Revista Ibero Americana de Ciências Ambientais, v.11, n.6, p.49-58, 2020. DOI: http://doi.org/10.6008/CBPC2179-6858.2020.006.0005 


\section{INTRODUÇÃO}

A compreensão dos fenômenos básicos da dinâmica dos atributos químicos e físicos do solo são importantes para a tomada de decisão, uma vez que o manejo do solo e das culturas geram mudanças no perfil do solo no sistema plantio direto que influenciam na dinâmica da acidez e da disponibilidade dos nutrientes e, por consequência, no manejo da fertilidade do solo (SANTOS et al., 2008).

Segundo Lana et al. (2015), a avaliação do solo é o primeiro passo para definir as medidas do manejo da fertilidade de um solo, tendo como ferramenta importante, a análise do solo, na avaliação dessa fertilidade ao diagnosticar os padrões físico-químicos do solo e, além disso, o conhecimento da fertilidade do solo e das necessidades nutricionais das plantas possibilita a identificação e quantificação dos nutrientes essenciais (BISSANI et al., 2004).

Conforme Zambolim et al. (2008), os sistemas agrícolas de produção dependem diretamente da qualidade física e química dos solos cultivados, assim como, das condições tecnológicas e climáticas do meio, entretanto, a qualidade ambiental também passou a ser fator limitante de produção, sendo comprometida pelo desequilíbrio e pela destruição dos meios naturais de regulação de dos sistemas.

O cultivo de culturas em sistemas integrados reflete positivamente na física e química do solo, visto que proporciona grande volume de raízes em profundidade, aumento da reciclagem de nutrientes, dos teores de matéria orgânica e de nutrientes no solo (CRUSCIOL et al., 2007). Portanto o bom funcionamento e boa qualidade do solo, principal meio de crescimento das plantas, é fundamental para garantir capacidade produtiva do agroecossistema e importante para a preservação de outros serviços essenciais, incluindo o fluxo da água, a biodiversidade e o equilíbrio de gases atmosféricos (DORAN et al., 1994).

Segundo Micheref et al. (2005), doenças radiculares estão entre as principais causas de redução na produtividade de culturas de interesse alimentar mundial. Dentre os organismos causadores de doenças radiculares destacam-se os fungos, as bactérias e os nematoides, denominados generalizadamente como patógenos radiculares ou fitopatógenos habitantes do solo. O controle de doenças radiculares é muito difícil, pois os patógenos co-evoluiram com as plantas por milhões de anos e estão altamente adaptados ao ambiente subterrâneo em associação com o hospedeiro.

Distúrbios como a "Soja louca II" faz com que a cultura da soja não produza vagens, o que a impede de concluir seu ciclo, caracterizada pela redução da produtividade em função do elevado índice de abortamento de flores e vagens e do alto percentual de desconto no valor da soja, devido à presença de impurezas, ou seja, pedaços de tecido verde e grãos podres, que propiciam apodrecimento da massa de grãos (MEDEIROS et al., 2016; MEYER et al., 2010).

Segundo Favoreto et al. (2015), a ocorrência de haste verde e retenção foliar na cultura da soja aumentou significativamente a partir da safra 2005/06, principalmente nos estados do Maranhão, Tocantins, Pará e norte do Mato Grosso. São estimadas reduções de produtividade de até $60 \%$, devido ao elevado índice de abortamento de flores e vagens e pela perda de qualidade dos grãos pela presença de impurezas. A incidência da SL II pode ocasionar perdas generalizadas inviabilizando a colheita em extensas áreas, mesmo 
após a dessecação com herbicidas (MEDEIROS et al., 2014).

Sob o ponto de vista agronômico essa anomalia é bastante preocupante, pois existe redução de produtividade e qualidade de grãos, dificuldade de colheita em virtude de talos verdes e, além disso, alongamento do período em que há folhas verdes de soja, aumentado a sobrevivência de fungos biotróficos, como a ferrugem asiática (BALBINOT JUNIOR, 2011).

Portanto, uma vez que a nutrição mineral das plantas, governada pela disponibilidade de nutriente no solo, tem sido um dos fatores mais estudados com relação à susceptibilidade e resistência de plantas a doenças (LUCAS, 1988; AGRIOS, 1988). Assim, qualquer modificação no solo pode alterar diretamente sua estrutura e sua atividade biológica e, consequentemente, sua fertilidade, com reflexos na qualidade ambiental e produtividade das culturas (BROOKES, 1995). Tornar-se necessário avaliar os atributos físico e químicos de solo onde ocorrem anomalias como a Soja Louca II, logo, o objetivo deste trabalho é avaliar os teores e variância dos atributos químicos de solos em áreas de com ocorrência da Soja Louca II.

\section{MATERIAIS E MÉTODOS}

O estudo foi realizado em 6 propriedades da Região do Planalto de Santarém-Belterra, que compreende os municípios de Santarém, Mojui do Campos e Belterra, localizados na Região Oeste do Estado do Pará o município de Santarém (Figura 2) com uma economia baseada na Indústria e Serviços tem seu setor agropecuária bem estabilizado, servindo como corredor de escoamento da produção de grãos do país pelo Porto da Cargil.

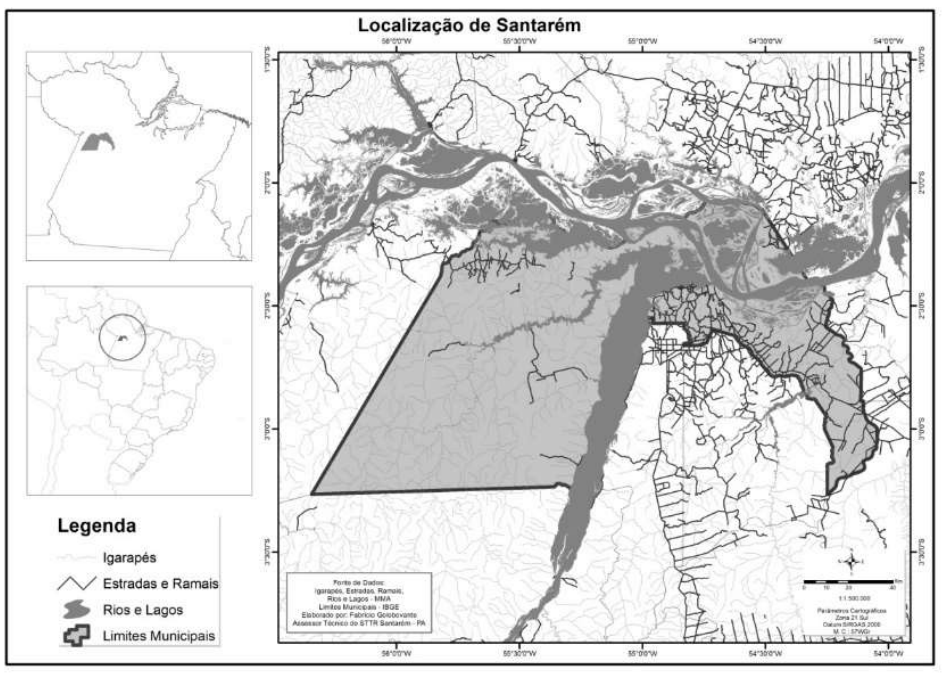

Figura 2: Mapa de localização de da área de estudo.

As propriedades são ecossistemas agrícolas com uma produção agrícola baseada no consórcio de soja e milho, são áreas antropizadas, modificadas pela ação humana para o desenvolvimento dos sistemas agrícolas de cultivo, o sistema de produção sistema plantio direto, com bastante mecanização agrícola e uso intensivo de produtos fitossanitários e fertilizantes químicos a pelo menos 10 anos de uso (Tabela 3) com predominância de Latossolo Amarelo Distrófico (EMBRAPA, 2016). 
Tabela 3: Local de coletas das Amostra de solo.

\begin{tabular}{llllll}
\hline Propriedade & Localização & Sistema de produção & Plano Safra & $\begin{array}{l}\text { Ano de } \\
\text { coleta }\end{array}$ & $\begin{array}{l}\text { Solo } \\
\text { Predominante }\end{array}$ \\
\hline Campo Experimental da Embrapa & Belterra & Plantio direto Soja X Milho & $2015 / 2016$ & 2016 & LATOSSOLO \\
\hline Fazenda Canarinho & Mojui do campos & Plantio direto Soja X Milho & $2015 / 2016$ & 2016 & LATOSSOLO \\
\hline Fazenda Pio & Belterra & Plantio direto Soja X Milho & $2015 / 2016$ & 2016 & LATOSSOLO \\
\hline Fazenda Horizonte I & Mojui do campos & Plantio direto Soja X Milho & $2015 / 2016$ & 2016 & LATOSSOLO \\
\hline Fazenda Esperança & Santarém & Plantio direto Soja X Milho & $2015 / 2016$ & 2016 & LATOSSOLO \\
\hline Fazenda Horizonte II- & Mojui do campos & Plantio direto Soja X Milho & $2015 / 2016$ & 2016 & LATOSSOLO \\
\hline
\end{tabular}

O clima da região está classificado no sistema Köppen como Ami, o qual pertence ao clima tropical, com características de clima quente e úmido apresentando temperaturas médias que oscilam entre a mínima de $25^{\circ} \mathrm{C}$ e máxima de $31^{\circ} \mathrm{C}$ e umidade relativa do ar em torno de $86 \%$, com dois períodos distintos um seco e o outro chuvoso (SILVA, 2011).

Foram coletadas 10 amostras simples de solo com auxílio de trado holandês, em 3 profundidades diferentes, 0 a 20 cm, 20-40 e 20 a 40 cm em Áreas: 1o Com ocorrência da Soja Louca-II na Safra 2015/2016 já demarcadas pelos produtores; 20 Sem ocorrência da anomalia safra 2015/2016 (Tabela 4).

As amostras de solo foram analisadas no Laboratório de Solo da Embrapa Amazônia Oriental em Belém-PA e determinado os teores dos atributos de solo potencial Hidrogeniônico $(\mathrm{pH})$, Fosforo $\left(\mathrm{P}^{+5}\right)$, Potássio $\left(\mathrm{K}^{+}\right)$, Sódio $\left(\mathrm{Na}^{+1}\right)$, Cálcio $\left(\mathrm{Ca}^{+2}\right)$ Magnésio $\left(\mathrm{Mg}^{+2}\right)$, Alumínio $\left(\mathrm{Al}^{+3}\right)$, Ferro $\left(\mathrm{Fe}^{+2}\right)$, Zinco $\left(\mathrm{Zn}^{+2}\right)$, Cobre $\left(\mathrm{Cu}^{+2},\right)$, Manganês $\left(\mathrm{Mn}^{+2}\right)$ e o teor de argila conforme os métodos adotados por EMBRAPA (2009). Os resultados foram submetidos à análise descritiva e à análise de variância ANOVA para dois critérios, Teste de Tukey a 5\%, comparando além da variação entre os tratamentos, a variabilidade entre os blocos, em um delineamento experimental de blocos ao acaso (VIEIRA, 2006), através do programa BioEstat versão 5.3.

Tabela 4: Método de coleta das amostras de solo.

TRATAMENTOS

Área de coleta

Sem ocorrência de SLII

Com ocorrência de SL-II na Safra 2015/2016.

\begin{tabular}{|c|c|c|c|}
\hline \multirow{3}{*}{ 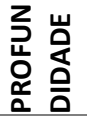 } & $0-20 \mathrm{~cm}$ & 10 amostras & 10 amostras \\
\hline & $20-40 \mathrm{~cm}$ & 10 amostras & 10 amostras \\
\hline & $40-60 \mathrm{~cm}$ & 10 amostras & 10 amostras \\
\hline
\end{tabular}

\section{RESULTADOS E DISCUSSÃO}

Os resultados da análise de solos em 20 com de profundidade, apresentados na Tabela 5, resultaram em níveis de acidez do solo, que se distribuem em Ph-4.5 alta acidez a Ph-5.8 baixa acidez (CRAVO et al., 2007), tanto em áreas com anomalia como em áreas sem ocorrência da "Soja Louca II". Solos com níveis de acidez elevados, fora da faixa adequado ( $\mathrm{pH}$ entre 6,0-6,5) para maior parte das culturas (MALAVOLTA, 2006), resultam na maior disponibilidade do Al tóxico e micronutrientes metálicos e menor disponibilidade de todos os macronutriente (CÂMARA, 2015).

Segundo Borkert et al. (1994), os altos teores de fósforo no solo podem induzir à deficiência de zinco desde que esses altos teores estejam associados com reduzidas absorção e translocação de $\mathrm{Zn}$, $\mathrm{Fe}$ e $\mathrm{Cu}$, porém os teores de $\mathrm{P}$ (Fosforo) nas amostras de solo variaram de 5 a $154 \mathrm{mg} / \mathrm{dm}^{3}$, apesar do alto coeficiente de variação os teores estão em sua maior parte abaixo dos recomentados para a cultura em Raij et al. (1996) e Cravo et al. (2007). Além disso a análise de variância para os teores $P$ (Tabela 6) não apresentaram diferença 
estatísticas significativa, entre as áreas com e sem ocorrência da Soja Louca II, para o Teste de TUKEY 5\%.

As plantas de Soja com deficiência de potássio (K) produzem grãos pequenos, enrugados e deformados e a maturidade da soja é atrasada, podendo causar também haste verde, retenção foliar e vagens chochas, Borkert et al. (1994), sintomas semelhantes ao da anomalia Soja louca-II descritos por Alves et al. (2014) e Medeiros et al. (2016), porém as amostras de solo em superfícies apresentaram teores classificados como médios e altos por Cravo et al. (2007) em solos do Estado Pará, descartando assim a possibilidades de deficiência de $\mathrm{K}$.

Vale ressaltar que a alta concentração de potássio tem ação antagonista sobre absorção de $\mathrm{Ca}^{2+} \mathrm{e}$ $\mathrm{Mg}^{2+}$, a diminuição na absorção cálcio pelo potássio deve-se à competição decorrente de propriedades fisiológicas destes cátions, enquanto a influência sobre a absorção do magnésio advém da competição por compostos ligantes do metabolismo do vegetal, ou seja evidenciando sintomas de deficiência desses nutrientes (FAGERIA, 2001).

Segundo Bergmann (1992) as plantas podem tolerar relativamente altas concentrações de potássio sem apresentar distúrbios. Porém, ele pode causar efeitos indiretos pela deficiência induzida de outros elementos, como o cálcio e o magnésio, além disso o excesso de potássio pode causar também a inibição na absorção de boro, zinco, manganês e amônio, induzindo, ou ao menos, contribuindo para a deficiência destes elementos. E em comum a deficiência dessas nutrientes causam redução no número de vagens, plantas de menor porte, encurtamento entre nós e baixa fecundação de flores (CÂMARA, 2015), todos esses sintomas são idênticos aos da Soja Louca II (ALVES et al., 2014; MEDEIROS et al., 2016).

Em todas amostras de solos os micronutrientes apresentaram classe de alta disponibilidade dos

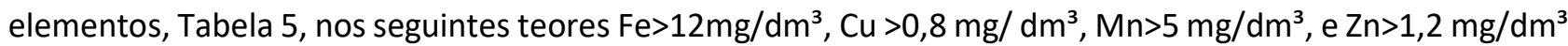
no solo, segundo as recomendações de Cravo et al. (2007), EMBRAPA (2011), Raij et al. (1996), SBCS (2004), Alvarez et al. (1999), Sousa et al. (2002), não havendo necessidade de correção nos teores de micronutrientes.

Porém a alta disponibilidade dos micronutriente metálicos, em decorrência do ph Baixo das amostras, pode ocasionar Fitotoxidez em plantas de soja (CÂMARA, 2015). O excesso de manganês (Mn) afeta mais diretamente a parte aérea do que as raízes com sintomas de toxicidade incluem uma clorose nas bordas dos folíolos seguida de necrose, com enrugamento por contração do folíolo e clorose das folhas novas, além de problemas fisiológicos específicos que são associados à toxicidade de manganês, que é o encarquilhamento dos folíolos (MALAVOLTA, 2006; BORKERT et al., 1994).

Com relação aos níveis tóxicos de Cobre (CU), o crescimento da soja é retardado e a cor da planta muda para verde acinzentado, verde azulado ou cor de oliva. Para muitas espécies de plantas, altas quantidades de cobre em solução nutritiva são tóxicas e limitam o crescimento, inclusive para a soja (MALAVOLTA, 2006; BORKERT et al., 1994; CÂMARA, 2015).

Foi observado os teores de $\mathrm{Zn}$ no solo do cerrado em níveis adequados de $0,3 \mathrm{mg} / \mathrm{kg}$ de solo e níveis tóxico de $33 \mathrm{mg} / \mathrm{kg}$ de solo para a soja enquanto, relatou os níveis críticos de $\mathrm{Zn}$ para a cultura de soja em solo de cerrado entre 0,27 a 0,37 mg kg-1 de solo, para solos do Estado do Para os níveis considerados altos 
de Zinco são de $Z n>2,2 \mathrm{mg} /$ dm$^{3}$ no solo, segundo as recomendações de Cravo et al. (2007). Um excesso de zinco pode ser tóxico nas plantas de soja, embora os níveis de tolerância sejam geralmente elevados podem induzir a deficiência de ferro.

Apesar da alta disponibilidade dos micronutrientes, as amostras de solo os não apresentaram diferença significativa entre os tratamentos com e sem ocorrência de soja louca II em nenhum dos atributos químico avaliados (Tabela 6). Ph e Zinco (Zn) são os atributos que apresentaram menor (7.45\%) e maior (198.17\%) Coeficiente de Variação, respectivamente.

Tabela 5: Resultados da análise de solo a $20 \mathrm{~cm}$ de profundidade.

\begin{tabular}{|c|c|c|c|c|c|c|c|c|c|c|c|c|c|c|c|}
\hline \multirow{2}{*}{$\begin{array}{l}\text { Atributos } \\
\text { Identifica } \\
\text { ção }\end{array}$} & \multirow[b]{2}{*}{ Lote } & \multirow[t]{2}{*}{$\begin{array}{l}\text { p } \\
\text { H. }\end{array}$} & $\mathbf{P}$ & $K$ & $\begin{array}{l}N \\
\mathrm{a}\end{array}$ & $\begin{array}{l}\text { C } \\
\text { a }\end{array}$ & $\begin{array}{l}\mathrm{Ca}+ \\
\mathrm{Mg}\end{array}$ & Al & $\mathrm{Fe}$ & $\mathrm{Zn}$ & $\mathrm{Cu}$ & $\mathrm{Mn}$ & SB & $\begin{array}{l}\text { CTC } \\
\text { (t) }\end{array}$ & \multirow{2}{*}{$\begin{array}{l}\begin{array}{l}\text { Teor } \\
\text { argila }\end{array} \\
\mathrm{g} / \mathrm{kg}\end{array}$} \\
\hline & & & \multicolumn{3}{|c|}{$\mathrm{mg} / \mathrm{dm}^{3}$} & \multicolumn{3}{|c|}{$\mathrm{cmolc} / \mathrm{dm}^{3}$} & \multicolumn{4}{|l|}{$\mathrm{mg} / \mathrm{kg}$} & \multicolumn{2}{|c|}{$\mathrm{cmolc} / \mathrm{dm}^{3}$} & \\
\hline \multirow{4}{*}{$\begin{array}{l}\text { Faz. } 1 \\
\text { Embrapa }\end{array}$} & Com & 5. & 10 & 60 & 2 & 3. & 4.8 & 0. & 650.2 & 21.0 & 2.75 & 287. & 4.9 & 5.06 & 140 \\
\hline & SL-II & 8 & & & & 9 & & 1 & 1 & 1 & & 43 & 6 & & \\
\hline & Sem & 5. & 56 & 41 & 7 & 4. & 5 & 0. & 526.1 & 24.2 & 3.43 & 166. & 5.1 & 5.24 & 160 \\
\hline & SLII & 6 & & & & 1 & & 1 & 3 & 6 & & 7 & 4 & & \\
\hline \multirow{4}{*}{$\begin{array}{l}\text { Faz.2 } \\
\text { Canarinho }\end{array}$} & Com & 5. & 154 & 21 & 2 & 2. & 3.9 & 0. & 1037. & 7.58 & 2.77 & 82.2 & 3.9 & 4.06 & 60 \\
\hline & SLII & 4 & & & & 7 & & 1 & 88 & & & 8 & 6 & & \\
\hline & Sem & 4. & 5 & 19 & 2 & 1. & 2 & 0. & 1296. & 7.36 & 1.65 & 66.0 & 2.0 & 2.16 & 60 \\
\hline & SLII & 5 & & & & 5 & & 1 & 37 & & & 2 & 6 & & \\
\hline \multirow{4}{*}{ Faz.3 Pio } & Com & 5. & 11 & 75 & 5 & 3. & 4.6 & 0. & 1089. & 36.6 & 7.14 & 228. & 4.8 & 4.91 & 220 \\
\hline & SLII & 4 & & & & 7 & & 1 & 55 & 4 & & 17 & 1 & & \\
\hline & Sem & 5. & 16 & 94 & 7 & 3. & 4.4 & 0. & 731.3 & 25.5 & 4.66 & 305. & 4.6 & 4.77 & 100 \\
\hline & SLII & 3 & & & & 7 & & 1 & 8 & 9 & & 94 & 7 & & \\
\hline \multirow{4}{*}{$\begin{array}{l}\text { Faz.4 } \\
\text { Horizonte } \\
\text { I - }\end{array}$} & Com & 4. & 9 & 80 & 2 & 3. & 3.9 & 0. & 0.37 & 9.3 & 36.6 & 2.79 & 4.2 & 4.92 & 40 \\
\hline & SLII & 6 & & & 6 & 3 & & 7 & & & 6 & & 2 & & \\
\hline & Sem & 5. & 11 & 10 & 2 & 2 & 2.4 & 0. & 0.63 & 6.2 & 53.9 & 1.98 & 2.7 & 3.17 & 20 \\
\hline & SLII & 3 & & 2 & 6 & & & 4 & & & 9 & & 7 & & \\
\hline \multirow{4}{*}{$\begin{array}{l}\text { Faz.5 } \\
\text { Esperança }\end{array}$} & Com & 5. & 23.3 & 10 & 4 & 3 & 3.8 & 0. & 1406. & 534. & 286. & 164. & 4.2 & 4.34 & 20 \\
\hline & SLII & 1 & & 6 & 0 & & & 1 & 63 & 99 & 42 & 81 & 4 & & \\
\hline & Sem & 5. & 28.2 & 97 & 3 & 3 & 3.7 & 0. & 788.3 & 444. & 136. & 289. & 4.1 & 4.21 & 220 \\
\hline & SLII & 2 & 2 & & 8 & & & 1 & 8 & 98 & 65 & 54 & 1 & & \\
\hline \multirow{4}{*}{$\begin{array}{l}\text { Faz. } 6 \\
\text { Horizente } \\
\text { II- }\end{array}$} & Com & 5. & 9 & 67 & 1 & 3. & 4.2 & 0. & 84.84 & 2.11 & 0.77 & 20.5 & 4.4 & 4.62 & 240 \\
\hline & SLII & 2 & & & 2 & 4 & & 2 & & & & 4 & 2 & & \\
\hline & Sem & 5. & 7 & 83 & 1 & 5. & 6.2 & 0. & 72.73 & 7.76 & 0.66 & 64.6 & 6.4 & 6.56 & 100 \\
\hline & SLII & 7 & & & 0 & 1 & & 1 & & & & 4 & 6 & & \\
\hline
\end{tabular}

Tabela 6: Análise descritiva e Análise de Variância dos atributos a $20 \mathrm{~cm}$ de profundidade.

\begin{tabular}{|c|c|c|c|c|c|c|c|c|c|c|c|}
\hline Atributos & $\mathbf{N}^{1}$ & Mín. & Máx. & $A^{2}$ & Média & $\mathrm{V}^{\mathbf{3}}$ & DP & CV & $\neq$ & $\mathbf{F}$ & (p) \\
\hline pH em agua. & 12 & 4.5 & 5.8 & 1.3 & 5.2583 & 0.1536 & 0.3919 & $7.45 \%$ & 0.08 & 0.1249 & 0.73 \\
\hline $\mathbf{P}$ & 12 & 5 & 154 & 149 & 28.2933 & 1765.373 & 42.0163 & $148.50 \%$ & 15.51 & 0.3862 & 0.5538 \\
\hline $\mathbf{K}$ & 12 & 19 & 106 & 87 & 70.4167 & 897.1742 & 29.9529 & $42.54 \%$ & 4.50 & 0.0619 & 0.8031 \\
\hline $\mathrm{Na}$ & 12 & 2 & 40 & 38 & 14.75 & 196.75 & 14.0268 & $95.10 \%$ & 0.50 & 0.0035 & 0.953 \\
\hline $\mathrm{Ca}$ & 12 & 1.5 & 5.1 & 3.6 & 3.2833 & 0.9124 & 0.9552 & $29.09 \%$ & 0.10 & 0.03 & 0.8599 \\
\hline $\mathrm{Ca}+\mathrm{Mg}$ & 12 & 2 & 6.2 & 4.2 & 4.075 & 1.2439 & 1.1153 & $27.37 \%$ & 0.25 & 0.1389 & 0.7168 \\
\hline Al & 12 & 0.1 & 0.7 & 0.6 & 0.1833 & 0.0342 & 0.185 & $100.93 \%$ & 0.07 & 0.367 & 0.5637 \\
\hline $\mathrm{Fe}$ & 12 & 0.37 & 1406.63 & 1406.26 & 640.425 & 260936.4 & 510.819 & $79.76 \%$ & 142.31 & 0.2163 & 0.6551 \\
\hline $\mathrm{Zn}$ & 12 & 2.11 & 534.99 & 532.88 & 93.9817 & 34687.22 & 186.245 & $198.17 \%$ & 15.91 & 0.02 & 0.8853 \\
\hline $\mathrm{Cu}$ & 12 & 0.66 & 286.42 & 285.76 & 44.7958 & 7362.906 & 85.8074 & $191.55 \%$ & 22.58 & 0.1925 & 0.6726 \\
\hline $\mathrm{Mn}$ & 12 & 1.98 & 305.94 & 303.96 & 140.07 & 13396.98 & 115.745 & $82.63 \%$ & 18.13 & 0.0674 & 0.7953 \\
\hline SB & 12 & 2.06 & 6.46 & 4.4 & 4.3183 & 1.2486 & 1.1174 & $25.88 \%$ & 0.23 & 0.1204 & 0.7344 \\
\hline CTC & 12 & 2.16 & 6.56 & 4.4 & 4.5017 & 1.1895 & 1.0906 & $24.23 \%$ & 10.00 & 0.2107 & 0.6591 \\
\hline Teor de argila & 12 & 20 & 240 & 220 & 115 & 6409.091 & 80.0568 & $69.61 \%$ & 0.30 & 0.0427 & 0.8342 \\
\hline
\end{tabular}

Os resultados de análise de solo, análise descritiva e de variância para a profundidade de $40 \mathrm{~cm}$ são 
apresentados na Tabela 7 e 8, onde os atributos não apresentarem diferença significativa entre as áreas com e sem de ocorrência da anomalia, com valores de F abaixo de 4,96 e valores (p) muito acima de 0,005, (VIEIRA, 2006), porem a analise descritiva dos atributos resultou em classe de acides ainda maiores que os encontrados a $20 \mathrm{~cm}$ de profundidades.

Conforme Schlindwein et al. (2000), altos teores de acidez em profundidade, ocorre devido a aplicação dos insumos é feita em linhas, na subsuperfície do solo, ou a lanço, na superfície, e a deposição superficial dos resíduos das culturas altera a taxa de decomposição da matéria orgânica e a liberação dos nutrientes na superfície do solo, ressaltando que níveis de acidez elevados, resultam na maior disponibilidade do Al tóxico e micronutrientes metálicos e menor disponibilidade de todos os macronutriente (MALAVOLTA, 2006),

Tabela 7: Resultados da análise de solo a $40 \mathrm{~cm}$ de profundidade.

\begin{tabular}{|c|c|c|c|c|c|c|c|c|c|c|c|c|c|c|c|}
\hline \multirow{2}{*}{$\begin{array}{l}\text { Atributos } \\
\text { Identificação }\end{array}$} & \multirow[b]{2}{*}{ Lote } & \multirow[t]{2}{*}{$\begin{array}{l}\mathrm{p} \\
\mathrm{H} .\end{array}$} & $P$ & K & $\begin{array}{l}\mathrm{N} \\
\mathrm{a}\end{array}$ & $\begin{array}{l}\mathrm{C} \\
\mathrm{a}\end{array}$ & $\begin{array}{l}\mathrm{Ca}+ \\
\mathrm{Mg}\end{array}$ & $\begin{array}{l}\text { A } \\
\text { I }\end{array}$ & $\mathrm{Fe}$ & $\mathrm{Zn}$ & $\mathrm{Cu}$ & $\mathrm{Mn}$ & SB & $\begin{array}{l}\text { CTc } \\
\text { efetiva } \\
\text { (t) }\end{array}$ & \multirow{2}{*}{$\begin{array}{l}\text { teor de } \\
\text { argila } \\
\mathrm{g} / \mathrm{kg}\end{array}$} \\
\hline & & & \multicolumn{3}{|c|}{$\mathrm{mg} / \mathrm{dm}^{3}$} & \multicolumn{3}{|c|}{$\mathrm{cmolc} / \mathrm{dm}^{3}$} & \multicolumn{4}{|c|}{$\mathrm{mg} / \mathrm{kg}$} & \multicolumn{2}{|c|}{$\mathrm{cmolc} / \mathrm{dm}^{3}$} & \\
\hline \multirow{4}{*}{$\begin{array}{l}\text { Faz. } 1 \text { Embrapa- } \\
\text { Belterra }\end{array}$} & Com & 4. & 4 & 2 & 2 & 1. & 1.7 & 0. & 401. & 4.4 & 2.1 & 47. & 1. & 2.36 & 20 \\
\hline & SL-II & 7 & & 1 & & 4 & & 6 & 8 & 7 & & 04 & 76 & & \\
\hline & Sem & 4. & 6 & 2 & 2 & 1. & 1.8 & 0. & 686. & 3.8 & 2.9 & 58. & 1. & 2.46 & 20 \\
\hline & SLII & 6 & & 1 & & 3 & & 6 & 64 & 3 & 2 & 56 & 86 & & \\
\hline \multirow{4}{*}{ Faz.2 Canarinho-Mojui } & Com & 4. & 31 & 9 & 2 & 0. & 0.7 & 1. & 519. & 2.4 & 2.1 & 14. & 0. & 1.93 & 20 \\
\hline & SLII & 4 & & & & 5 & & 2 & 98 & 2 & & 34 & 73 & & \\
\hline & Sem & 4. & 2 & 1 & 2 & 0. & 0.8 & 1. & 1158 & 0.2 & 1.5 & 29. & 0. & 2.24 & 20 \\
\hline & SLII & 2 & & 3 & & 6 & & 4 & .18 & 8 & 6 & 27 & 84 & & \\
\hline \multirow{4}{*}{ Faz.3 Pio- Belterra } & Com & 4. & 5 & 3 & 2 & 1. & 1.8 & 0. & 874. & 7.4 & 4.1 & 42. & 1. & 2.50 & 20 \\
\hline & SLII & 8 & & 6 & & 5 & & 6 & 8 & 8 & 7 & 05 & 90 & & \\
\hline & Sem & 5 & 5 & 3 & 5 & 2. & 2.9 & 0. & 604. & 7.0 & 3.0 & 82. & 3. & 3.62 & 20 \\
\hline & SLII & & & 8 & & 5 & & 6 & 49 & 1 & 9 & 91 & 02 & & \\
\hline \multirow{4}{*}{$\begin{array}{l}\text { Faz.4 Horizonte I- } \\
\text { Mojui dos Campos }\end{array}$} & Com & 5. & 5 & 5 & 3 & 2. & 2.9 & 0. & 39.3 & 1.7 & 0.3 & 6.5 & 3. & 3.39 & 60 \\
\hline & SLII & 4 & & 9 & 1 & 3 & & 2 & 2 & 2 & 4 & 8 & 19 & & \\
\hline & Sem & 5. & 8 & 7 & 3 & 1. & 1.6 & 0. & 64.5 & 1.0 & 0.4 & 9.0 & 1. & 2.54 & 20 \\
\hline & SLII & 1 & & 5 & 5 & 2 & & 6 & 7 & 9 & & 1 & 94 & & \\
\hline \multirow{4}{*}{$\begin{array}{l}\text { Faz.5 Esperança - } \\
\text { Santarém }\end{array}$} & Com & 4. & 6. & 6 & 4 & 1. & 1.7 & 0. & 742. & 456 & 378 & 44. & 2. & 2.64 & 20 \\
\hline & SLII & 9 & 7 & 0 & 2 & 3 & & 6 & 57 & .44 & .5 & 96 & 04 & & \\
\hline & Sem & 4. & 6. & 4 & 3 & 1 & 1.3 & 0. & 679 & 326 & 142 & 77. & 1. & 2.16 & 20 \\
\hline & SLII & 9 & 44 & 4 & 5 & & & 6 & & .06 & .36 & 8 & 56 & & \\
\hline \multirow{4}{*}{$\begin{array}{l}\text { Faz. } 6 \text { Horizente II- } \\
\text { Mojui dos Campos }\end{array}$} & & 4. & 4 & 3 & 7 & 1. & 1.9 & 0. & 96.5 & 2.1 & 0.7 & 5.7 & 2. & 2.62 & 20 \\
\hline & & 7 & & 4 & & 6 & & 6 & 7 & 1 & 7 & 8 & 02 & & \\
\hline & Sem & 5 & 3 & 5 & 7 & 1. & 2.3 & 0. & 78.0 & 0.9 & 0.5 & 9.6 & 2. & 2.66 & 20 \\
\hline & SLII & & & 0 & & 9 & & 2 & 5 & 5 & & 5 & 46 & & \\
\hline
\end{tabular}

Segundo a classificação de Cravo et al. (2007), os teores dos atributos químicos das amostras à 40cm de profundidade são classificados como, $\mathrm{pH}$ muito ácido, $\mathrm{P}, \mathrm{K}, \mathrm{Na}, \mathrm{Ca}, \mathrm{Ca}+\mathrm{Mg}, \mathrm{Al}$ como baixo e micronutrientes alto em excesso e, ambos os tratamentos. Os atributos com menor $(6,66 \%)$ e maior $(250,70 \%)$ coeficiente de variação são pH e Cu respectivamente. Ressaltando que a toxidez causada por Cobre (Cu) causa sintomas idênticos aos de Soja Louca II, retardando o crescimento da soja o e mudando a cor da planta para verde acinzentado, verde azulado ou cor de oliva (MALAVOLTA, 2006; CÂMARA, 2015).

Tabela 8: Análise descritiva e análise de variância dos atributos à $40 \mathrm{~cm}$ de profundidade.

\begin{tabular}{llllllllllll}
\hline Atributos & $\mathbf{N}^{\mathbf{1}}$ & Mín & Máx & $\mathbf{A}^{\mathbf{2}}$ & Média & $\mathbf{V}^{\mathbf{3}}$ & $\mathbf{D P}$ & $\mathbf{C V}$ & $\boldsymbol{\#}$ & $\mathbf{F}$ & $\mathbf{( p )}$ \\
\hline pH em agua & 12 & 4.2 & 5.4 & 1.2 & 4.8083 & 0.1027 & 0.3204 & $6.66 \%$ & 0.0167 & 0.0074 & 0.9308 \\
\hline P & 12 & 2 & 31 & 29 & 7.1783 & 59.002 & 7.6813 & $107.01 \%$ & 4.21 & 0.8924 & 0.6303 \\
\hline K & 12 & 9 & 75 & 66 & 38.3333 & 412.4242 & 20.3082 & $52.98 \%$ & 3.6667 & 0.0897 & 0.7671 \\
\hline
\end{tabular}




\begin{tabular}{llllllllllll}
\hline $\mathrm{Na}$ & 12 & 2 & 42 & 40 & 14.3333 & 259.333 & 16.1038 & $112.35 \%$ & 0 & 0 & 0.9955 \\
\hline $\mathrm{Ca}$ & 12 & 0.5 & 2.5 & 2 & 1.425 & 0.362 & 0.6017 & $42.22 \%$ & 0.0167 & 0.0021 & 0.9634 \\
\hline $\mathrm{Ca}+\mathrm{Mg}$ & 12 & 0.7 & 2.9 & 2.2 & 1.7833 & 0.4724 & 0.6873 & $38.54 \%$ & 0 & 0 & 0.9955 \\
\hline $\mathrm{Al}$ & 12 & 0.2 & 1.4 & 1.2 & 0.65 & 0.1173 & 0.3425 & $52.68 \%$ & 0.0333 & 0.0259 & 0.8695 \\
\hline $\mathrm{Fe}$ & 12 & 39.32 & 1158.18 & 1118.86 & 495.4975 & 132975.6 & 364.658 & $73.59 \%$ & 99.315 & 0.2065 & 0.6622 \\
\hline $\mathrm{Zn}$ & 12 & 0.28 & 456.44 & 456.16 & 67.8217 & 23600.95 & 153.626 & $226.51 \%$ & 22.57 & 0.0592 & 0.8071 \\
\hline $\mathrm{Cu}$ & 12 & 0.34 & 378.5 & 378.16 & 44.9008 & 12671.22 & 112.567 & $250.70 \%$ & 39.525 & 0.3479 & 0.5738 \\
\hline $\mathrm{Mn}$ & 12 & 5.78 & 82.91 & 77.13 & 35.6625 & 764.5597 & 27.6507 & $77.53 \%$ & 17.7417 & 1.2648 & 0.2871 \\
\hline $\mathrm{SB}$ & 12 & 0.73 & 3.19 & 2.46 & 1.9433 & 0.534 & 0.7308 & $37.60 \%$ & 0.0067 & 0.0002 & 0.9854 \\
\hline $\mathrm{CTC}$ & 12 & 1.93 & 3.62 & 1.69 & 2.5933 & 0.2302 & 0.4798 & $18.50 \%$ & 6.6667 & 1 & 0.3427 \\
\hline Teor de argila & 12 & 20 & 60 & 40 & 23.3333 & 133.3333 & 11.547 & $49.49 \%$ & 0.04 & 0.019 & 0.8881 \\
\hline
\end{tabular}

${ }^{1}$ Numero de Amostras

${ }^{2}$ Amplitude

${ }^{3}$ Variância

Tabela 9: Tabela 7: Resultados da análise de solo a $60 \mathrm{~cm}$ de profundidade.

\begin{tabular}{|c|c|c|c|c|c|c|c|c|c|c|c|c|c|c|c|}
\hline Atributos & & $\begin{array}{l}\mathrm{pH} \\
.\end{array}$ & $P$ & K & $\mathrm{Na}$ & $\mathrm{Ca}$ & $\begin{array}{l}\mathrm{Ca}+ \\
\mathrm{Mg}\end{array}$ & Al & $\mathrm{Fe}$ & $\mathrm{Zn}$ & $\mathrm{Cu}$ & $\mathrm{Mn}$ & SB & $\begin{array}{l}\text { CTc } \\
\text { efetiva } \\
\text { (t) }\end{array}$ & $\begin{array}{l}\text { teor de } \\
\text { argila }\end{array}$ \\
\hline \multirow[t]{2}{*}{ Identificação } & & ag & \multirow{2}{*}{\multicolumn{3}{|c|}{$\mathrm{mg} / \mathrm{dm}^{3}$}} & \multirow{2}{*}{\multicolumn{3}{|c|}{$\mathrm{cmolc} / \mathrm{dm}^{3}$}} & \multirow{2}{*}{\multicolumn{4}{|c|}{$\mathrm{mg} / \mathrm{kg}$}} & \multirow{2}{*}{\multicolumn{2}{|c|}{$\mathrm{cmolc} / \mathrm{dm}^{3}$}} & $\mathrm{~g} / \mathrm{kg}$ \\
\hline & Lote & ua & & & & & & & & & & & & & \\
\hline \multirow{4}{*}{$\begin{array}{l}\text { Faz. } 1 \text { Embrapa- } \\
\text { Belterra }\end{array}$} & Com & 4. & 2. & 13. & 2.0 & 1. & 1.30 & 0. & 260 & 20. & 1.5 & 25. & 1. & 1.94 & 20.00 \\
\hline & SL-II & 60 & 00 & 00 & 0 & 00 & & 60 & .52 & 61 & 1 & 38 & 34 & & \\
\hline & Sem & 4. & 3. & 15. & 2.0 & 1. & 1.40 & 0. & 509 & 1.3 & 2.1 & 26. & 1. & 2.05 & 40.00 \\
\hline & SLII & 50 & 00 & 00 & 0 & 10 & & 60 & .25 & 4 & 8 & 33 & 45 & & \\
\hline \multirow{4}{*}{$\begin{array}{l}\text { Faz.2 Canarinho- } \\
\text { Mojui dos }\end{array}$} & Com & 4. & 2. & 11. & 2.0 & 0. & 0.60 & 1. & 417 & 0.3 & 1.9 & 11. & 0. & 1.94 & 20.00 \\
\hline & SLII & 20 & 00 & 00 & 0 & 40 & & 30 & .26 & 0 & 2 & 60 & 64 & & \\
\hline & Sem & 4. & 2. & 9.0 & 2.0 & 0. & 0.90 & 1. & 751 & 1.5 & 1.7 & 20. & 0. & 1.93 & 20.00 \\
\hline & SLII & 30 & 00 & 0 & 0 & 70 & & 00 & .37 & 3 & 0 & 38 & 93 & & \\
\hline \multirow[t]{4}{*}{ Faz.3 Pio- Belterra } & Com & 4. & 5. & 34. & 23. & 1. & 1.50 & 0. & 643 & 2.8 & 3.9 & 31. & 1. & 2.29 & 20.00 \\
\hline & SLII & 70 & 00 & 00 & 00 & 20 & & 60 & .34 & 3 & 7 & 32 & 69 & & \\
\hline & Sem & 4. & 5. & 36. & 2.0 & 1. & 1.70 & 0. & 352 & 6.1 & 2.3 & 34. & 1. & 2.40 & 20.00 \\
\hline & SLII & 70 & 00 & 00 & 0 & 40 & & 60 & .82 & 5 & 0 & 62 & 80 & & \\
\hline \multirow{4}{*}{$\begin{array}{l}\text { Faz.4 Horizonte I - } \\
\text { Mojui dos }\end{array}$} & Com & 5. & 3. & 45. & 28. & 1. & 2.20 & 0. & 26. & 0.8 & 0.4 & 2.4 & 2. & 2.64 & 0.00 \\
\hline & SLII & 20 & 00 & 00 & 00 & 50 & & 20 & 10 & 6 & 6 & 5 & 44 & & \\
\hline & Sem & 4. & 4. & 59. & 28. & 0. & 1.00 & 0. & 39. & 0.6 & 0.2 & 3.9 & 1. & 1.97 & 20.00 \\
\hline & SLII & 90 & 00 & 00 & 00 & 80 & & 70 & 42 & 3 & 7 & 4 & 27 & & \\
\hline \multirow{4}{*}{$\begin{array}{l}\text { Faz.5 Esperança - } \\
\text { Santarém }\end{array}$} & Com & 4. & 4. & 42. & 40. & 0. & 1.10 & 0. & 662 & 338 & 464 & 31. & 1. & 1.98 & 20.00 \\
\hline & SLII & 80 & 40 & 00 & 00 & 80 & & 60 & .85 & .69 & .08 & 35 & 38 & & \\
\hline & Sem & 4. & 3. & 30. & 35. & 0. & 0.90 & 0. & 355 & 484 & 258 & 25. & 1. & 1.83 & 40.00 \\
\hline & SLII & 80 & 67 & 00 & 00 & 60 & & 70 & .58 & .73 & .57 & 43 & 13 & & \\
\hline \multirow{4}{*}{$\begin{array}{l}\text { Faz. } 6 \text { Horizente II- } \\
\text { Mojui dos }\end{array}$} & Com & 4. & 3. & 23. & 7.0 & 1. & 1.90 & 0. & 38. & 0.2 & 0.3 & 1.9 & 1. & 2.59 & 20.00 \\
\hline & SLII & 50 & 00 & 00 & 0 & 00 & & 60 & 97 & 2 & 2 & 8 & 99 & & \\
\hline & Sem & 4. & 3. & 30. & 7.0 & 0. & 1.10 & 0. & 48. & 0.3 & 0.3 & 2.5 & 1. & 1.81 & 20.00 \\
\hline & SLII & 50 & 00 & 00 & 0 & 90 & & 60 & 84 & 7 & 9 & 0 & 21 & & \\
\hline
\end{tabular}

Em $60 \mathrm{~cm}$ de profundidade os atributos repetem a classificação de teores proposta por Cravo et.al. (2007) para solos da região, em teores dos atributos químicos das são classificados como, pH muito ácido, P, K, Na, Ca, Ca+Mg, Al como baixo e micro nutrientes alto em excesso e, ambos os tratamentos. Condições ideais, com teores de Potássio críticos e Micronutrientes em níveis tóxicos, para manifestação de sintomas idênticos aos da Soja Louca II (CÂMARA, 2015; ALVES et al., 2014; MEDEIROS et al., 2016).

Tabela 10: Análise descritiva e Análise de Variância dos atributos a $60 \mathrm{~cm}$ de profundidade.

\begin{tabular}{llllllllllll}
\hline Atributos & $\mathbf{N}^{\mathbf{1}}$ & $\mathbf{M i ́ n}$ & $\mathbf{M a ́ x}$ & $\mathbf{A}^{\mathbf{2}}$ & $\mathbf{M e ́ d i a}$ & $\mathbf{V}^{\mathbf{3}}$ & $\mathbf{D P}$ & $\mathbf{C V}$ & $\boldsymbol{\#}$ & $\mathbf{F}$ & $\mathbf{( p )}$ \\
\hline $\mathbf{p H}$ em agua & 12 & 4.2 & 5.2 & 1 & 4.6417 & 0.0736 & 0.2712 & $5.84 \%$ & 0.05 & 0.0936 & 0.7626 \\
\hline $\mathbf{P}$ & 12 & 2 & 5 & 3 & 3.3392 & 1.1844 & 1.0883 & $32.59 \%$ & 0.2117 & 0.1042 & 0.7509 \\
\hline $\mathbf{K}$ & 12 & 9 & 59 & 50 & 28.9167 & 237.5379 & 15.4123 & $53.30 \%$ & 1.8333 & 0.0387 & 0.8417 \\
\hline $\mathbf{N a}$ & 12 & 2 & 40 & 38 & 14.8333 & 218.1515 & 14.77 & $99.57 \%$ & 4.3333 & 0.2404 & 0.6384 \\
\hline $\mathbf{C a}$ & 12 & 0.4 & 1.5 & 1.1 & 0.95 & 0.1027 & 0.3205 & $33.74 \%$ & 0.0667 & 0.1194 & 0.7353 \\
\hline $\mathbf{C a}+\mathbf{M g}$ & 12 & 0.6 & 2.2 & 1.6 & 1.3 & 0.2145 & 0.4632 & $35.63 \%$ & 0.2667 & 0.9938 & 0.6561 \\
\hline $\mathbf{A l}$ & 12 & 0.2 & 1.3 & 1.1 & 0.675 & 0.0693 & 0.2633 & $39.00 \%$ & 0.05 & 0.0993 & 0.7562 \\
\hline
\end{tabular}




\begin{tabular}{llllllllllll}
\hline Fe & 12 & 26.1 & 751.37 & 725.27 & 342.1933 & 70093.49 & 264.752 & $77.37 \%$ & 1.3733 & 0.0001 & 0.9895 \\
\hline Zn & 12 & 0.22 & 484.73 & 484.51 & 71.5217 & 26251.46 & 162.023 & $226.54 \%$ & 21.8733 & 0.05 & 0.8216 \\
\hline Cu & 12 & 0.27 & 464.08 & 463.81 & 61.4725 & 21537.95 & 146.758 & $238.74 \%$ & 34.475 & 0.1528 & 0.7046 \\
\hline Mn & 12 & 1.98 & 34.62 & 32.64 & 18.1067 & 163.072 & 12.77 & $70.53 \%$ & 1.52 & 0.0388 & 0.8416 \\
\hline SB & 12 & 0.64 & 2.44 & 1.8 & 1.4392 & 0.2351 & 0.4849 & $33.69 \%$ & 0.2817 & 1.0135 & 0.3395 \\
\hline CTC & 12 & 1.81 & 2.64 & 0.83 & 2.1142 & 0.0842 & 0.2902 & $13.73 \%$ & 10 & 3.4615 & 0.0888 \\
\hline teor de argila & 12 & 0 & 40 & 40 & 21.6667 & 106.0606 & 10.2986 & $47.53 \%$ & 0.2317 & 2.1039 & 0.1753 \\
\hline
\end{tabular}

${ }^{1}$ Numero de Amostras

${ }^{2}$ Amplitude

${ }^{3}$ Variância

Os Atributos não apresentaram diferença significativa entre os tratamentos com e sem Soja Louca-II em $60 \mathrm{~cm}$ de profundidade para o Teste de Tukey $5 \%$. A análise de variância dos atributos químicos não apresentou diferenças significativa entre as profundidades.

\section{CONCLUSÕES}

As áreas com os atributos avaliados não apresentarem diferença significativa entre os tratamentos, com valores de $\mathrm{F}$ abaixo de 4,96 e valores ( $p$ ) muito acima de 0,005 , sendo consideradas estatisticamente semelhantes as áreas com e sem de ocorrência da anomalia. As altas taxa de micronutrientes podem refletir sintomas semelhantes aos da Soja louca II assim como os teores de Potássio, que variaram de altos a críticos conforme a profundidade aumenta, porém ambas os tratamentos apresentaram altos níveis de micronutriente anulando a hipótese de fitotoxidez causadas por micronutrientes e por sua vez, desconsiderando, nesta situação, a hipótese que a anomalia pode ser causada pela deficiência ou excesso dos atributos avaliados.

\section{REFERÊNCIAS}

AGRIOS, G. N.. Plant disease epidemiology. In: Plant Pathology. 3 ed. Academic Press INC., 1988.

ALVES, L. W. R.; SILVA, S. R.; CASTRO, G. S. A.. Avaliação das propriedades físicas do solo e ocorrência da anomalia "Soja louca II" em sistemas de produção agrícola na Amazônia. Macapá: Embrapa Amapá; Belém: Embrapa Amazônia Oriental, 2014.

ALVAREZ, V. V. H.; NOVAIS, R. F.; BARROS, N. F.; CANTARUTTI, R. B.; LOPES, A. S.. Interpretação dos resultados das análises de solos. In: RIBEIRO, A. C.; GUIMARAES, P. T. G.; ALVAREZ, V. V. H.. Recomendações para uso de corretivo e Fertilizantes em Minas Gerais. Viçosa: CFSEMG, 1999. p.25-32.

BALBINOT JUNIOR, A. A.. Soja louca 2. Canoinhas: Correio do Norte, 2011.

BERGMANN, W.. Nutritional disorders of plants: development, visual and analytical diagnosis. New York: Gustav Fischer Publishing House Jena, 1992.

BISSANI, C. A.; GIANELLO, C.; TEDESCO, M. J.; CAMARGO, F. A. O.. Fertilidade dos solos e manejo da adubação das culturas. Porto Alegre: Gênesis, 2004.
BORKERT, C. M.; YORINORI, J. T.; CORRÊA-FERREIRA, B. S.; ALMEIDA, A. M. R.; FERREIRA, L. P.; SFREDO, G. J.. Seja o doutor da sua soja. Informações agronômicas, 1994.

BROOKES, P. C.. The use of microbial parameters in monitoring soil pollution by heavy metals. Biol. Fert. Soils, v.19, p.269-279, 1995.

CÂMARA, G. M. S.. Adubação. In: SILVA, F.; BORÉM, A.; SEDIYAMA, T.. Soja: do plantio à colheita. Viçosa: UFV, 2015. p.110-148.

CRAVO, M. S.; VIÉGAS, I. J. M.; BRASIL, E. C.. Recomendações de adubação e calagem para o estado do Pará. Belém: Embrapa Amazônia Oriental, 2007.

CRUSCIOL, C. A. C.; BORGHI, E.. Consórcio de milho com braquiária: produção de forragem e palhada para o plantio direto. Revista Plantio Direto, Passo Fundo, v.16, n.100, p.10-14, 2007.

DORAN, J. W.; PARKIN, T. B.. Defining and assessing soil quality. In: SYMPOSIUM DEFINING SOIL QUALITY FOR A SUSTAINABLE ENVIRONMENT. Anais. Minneapolis, 1994. p.3-21. 
EMBRAPA. Empresa Brasileira de Pesquisa Agropecuária. Manual de análises químicas de solos, plantas e fertilizantes. 2 ed. Brasília: EMBRAPA, 2009.

EMBRAPA. Empresa Brasileira de Pesquisa Agropecuária. Mapas de Solos e de Aptidão Agrícola das Áreas Alteradas do Pará. 500 exemplares. Belém: EMBRAPA, 2016.

FAGERIA, V. D.. Nutrient interacions in crop plants. Journal of Plant Nutrition, New York, v.24, p.1269-1290, 2001.

FAVORETO, L.; MEYER, M. C.; KLEPKER, K.; CAMPOS, L. J. M.. Ocorrência de Aphelenchoides sp. em plantas de soja com sintomas de Soja Louca II. In: CONGRESSO BRASILEIRO DE NEMATOLOGIA, 32. Anais. Londrina, 2015. p.81-82.

LUCAS, J. A.. Plant pathology and plant pathogens. 3 ed. Blackwell Science Ltd., 1998.

LANA, R. M. Q.; VASCONCELOS, A. C. P.; SILVA, A. A.; LIMA, L. C.; OLIVEIRA, L. R. R.. Análise descritiva dos atributos químicos do solo de propriedades rurais. Enciclopédia Biosfera, Goiânia, v.11 n.21, p.98, 2015.

MALAVOLTA, E.. Manual de nutrição de plantas. São Paulo: Agronômica Ceres, 2006.

MEDEIROS, S. R.; CARVALHO, E. A.; CONCEIÇÃO, V. D.; CANCIAN, E.. Diagnóstico precoce da 'soja louca II' no estado do Pará. In: SEMINÁRIO ANUAL DE INICIAÇÃO CIENTÍFICA DA UFRA, 12. Anais. Belém, 2014.

MEDEIROS, S. R.; CARVALHO, E.. A Incidência da Soja Louca II nos Sistemas Plantio Direto e Convencional. Comunicado Técnico 278. Belém: Embrapa Amazônia Oriental, 2016.

MEYER, M. C.; ALMEIDA, A. M. R.; GAZZIERO, D. L. P.; LIMA, D.. Soja louca II: Um problema de causa desconhecida. Embrapa Soja, 2010.
MICHEREF, S. J.; ANDRADE D. E. G. T.; MENEZES, M. Ecologia e manejo de patógenos radiculares em solos tropicais. Recife: UFRPE, 2005.

RAIJ, B. V.; SILVA, N. M.; BATAGLIA, O. C.; CANTARELLA, H.; QUAGGIO, J. A.; FURLANI, A. M. C.. Recomendações de adubação e calagem para o Estado de São Paulo. 2 ed. Campinas: Instituto Agronômico \& Fundação IAC, 1996.

SANTOS, D. R.; GATIBONI, L. C.; KAMINSKI, J.. Fatores que afetam a disponibilidade do fósforo e o manejo da adubação fosfatada em solos sob sistema plantio direto. Ciência Rural, v.38, n.2, 2008

SBCS. Sociedade brasileira de ciência do solo. Comissão de química e fertilidade do solo. Manual de adubação e calagem para os Estados do Rio grande do Sul e Santa Catarina. 10 ed. Porto Alegre: SBCS, 2004.

SCHLINDWEIN, J. A.; ANGHINONI, I.. Variabilidade vertical de fósforo e potássio disponíveis e profundidade de amostragem do solo no sistema plantio direto. Ciência Rural, Santa Maria, v.30, n.4, p.611-617, 2000.

SILVA, B. E. B.. Análise da tendência de aumento da precipitação anual na região de Santarém (PA), entre 1961 e 2008. In: SIMPÓSIO INTERNACIONAL DE CLIMATOLOGIA, 4. Anais. João Pessoa: SBMET, 2011. p.2-6.

SOUSA, D. M. G.; LOBATO, E.. Cerrado: correção de solo e adubação. Planaltina, Embrapa Cerrados, 2002.

VIEIRA, S.. Analise de Variância: Anova. São Paulo: Atlas, 2006.

ZAMBOLIM, L.; PICANÇO, M. C.; SILVA, A. A.; FERREIRA, L. R.; FERREIRA, F. A.. Produtos Fitossanitarios (Fungicidas, inseticidas, acaricidas e Herbicidas). Viçosa: UFV, 2008.

A CBPC - Companhia Brasileira de Produção Científica (CNPJ: 11.221.422/0001-03) detém os direitos materiais desta publicação. Os direitos referem-se à publicação do trabalho em qualquer parte do mundo, incluindo os direitos às renovações, expansões e disseminações da contribuição, bem como outros direitos subsidiários. Todos os trabalhos publicados eletronicamente poderão posteriormente ser publicados em coletâneas impressas sob coordenação da Sustenere Publishing, da Companhia Brasileira de Produção Científica e seus parceiros autorizados. Os (as) autores (as) preservam os direitos autorais, mas não têm permissão para a publicação da contribuição em outro meio, impresso ou digital, em português ou em tradução. 